\title{
Association of TSC gene variants and hypertension in Mongolian and Han populations
}

\author{
P.Y. Chang ${ }^{1}$, L.G. Zhao ${ }^{2}$ and X.L. Su ${ }^{1,3}$ \\ ${ }^{1}$ Department of Cell Biology, Capital Medical University, Beijing, China \\ ${ }^{2}$ Department of Preventive Medicine, \\ Inner Mongolia Medical College, Huhhot, Inner Mongolia, China \\ ${ }^{3}$ Clinical Medical Research Center, Department of Surgery, \\ Inner Mongolia Medical College Affiliated Hospital, Inner Mongolia, China \\ Corresponding author: X.L. Su \\ E-mail: xlsu@hotmail.com
}

Genet. Mol. Res. 10 (2): 902-909 (2011)

Received December 22, 2010

Accepted January 31, 2011

Published May 17, 2011

DOI 10.4238/vol10-2gmr1227

\begin{abstract}
We investigated a possible association between genetic variations in the thiazide-sensitive $\mathrm{Na}-\mathrm{Cl}$ cotransporter (TSC) gene and essential hypertension (EH) in the Mongolian and Han ethnic groups in Inner Mongolia. Our study included 385 unrelated Mongolian herdsmen and 523 Han farmers. Nine tagSNPs of TSC were identified from the Chinese HapMap database based on pairwise $\mathrm{r}^{2} \geq 0.5$ and minor allele frequency $\geq 0.05$. Genotyping was performed using the PCR/ligase detection reaction assay. Association between tagSNPs and hypertension was investigated under the additive model. There were significant differences between the genotype and allele frequencies of rs 13306673 between the EH group and the control group in the Han population. Significant associations were found between the rs 7204044 variant and $\mathrm{EH}$ in both the Mongolian and Han ethnic groups. The frequency of haplotype GCA in the EH group was significantly higher than in the control group in the Mongolian population. In the Han population, the frequency of haplotype TGG was significantly higher in the EH group than in controls, whereas haplotype TGA occurred significantly less often in EH than in controls. We suggest that rs7204044 of TSC is a
\end{abstract}


genetic factor for $\mathrm{EH}$ in these two ethnicities and that rs 13306673 is a genetic factor for $\mathrm{EH}$ in the Han population.

Key words: Essential hypertension; Mongolian population; TSC; Single nucleotide polymorphism

\section{INTRODUCTION}

Hypertension is a multifactorial disorder resulting from complex interactions between genetic and environmental contributions. A body of evidence suggests that the thiazide-sensitive $\mathrm{Na}-\mathrm{Cl}$ cotransporter (TSC) plays an important role in the development and progression of hypertension. The TSC gene, consisting of 26 exons that encode 1021 -amino acid residues, is located on chromosome $16 \mathrm{q} 13$. TSC has been reported to play a major role in sodium chloride reabsorption in the distal convoluted tubule (Plotkin et al., 1996), which is responsible for the reabsorption of 5-10\% of the filtered sodium chloride (Obermüller et al., 1995) and accounts for a significant portion of total renal sodium reabsorption. Mutations in the human TSC gene affect blood pressure regulation, as seen in Gitelman syndrome, a disease characterized by sodium wasting and low blood pressure (Simon et al., 1996). This cotransporter is the target of thiazide diuretics used to treat high blood pressure.

Recently, Melander et al. (2000) found a novel TSC gene molecular variant, A2736, which they found to be associated with hypertension. A2736 homozygotes were over-represented in hypertensive patients compared with control subjects, suggesting that the A2736 variant increased the risk for developing essential hypertension (EH) in southern Sweden. However, the association of this variant remains inconclusive with confirmation or lack thereof in several follow-up studies conducted in populations of diverse ethnicities (Matsuo et al., 2004; Keszei et al., 2007; Zhan et al., 2007; Wang et al., 2008). Analysis of a single polymorphism could be perceived as a limitation in assessing genetic association of a putative locus. To guard against this potential limitation we conducted a comprehensive association analysis of common tagSNPs (single nucleotide polymorphisms) of the TSC gene in a haplotype analysis of the Mongolian and Han ethnic groups in Inner Mongolia.

\section{MATERIAL AND METHODS}

\section{Subjects}

We recruited subjects aged 20-75 years from two villages in Duolun and three villages in Erenhot of Xilin Gol League in Inner Mongolia. Subjects with a history of secondary hypertension, stroke, coronary heart disease, diabetes, kidney failure, thyroid gland disease, or excessive drinking were excluded from this study. A total of 385 unrelated Mongolian herdsmen and 523 Han farmers were enrolled, including 158 Mongolian EH patients, 227 Mongolian normotensives (controls), 284 Han EH patients, and 239 Han normotensives (controls). Each subject was from a family that had been living in Inner Mongolia for at least three generations without a history of mixed marriage. We adopted the WHO's 1999 definition of hypertension in this study: a systolic blood pressure (SBP) of at least $140 \mathrm{mmHg}$ and/or diastolic blood pressure (DBP) of at least $90 \mathrm{mmHg}$ or antihypertension treatment. The normotensive group was selected based on the following criteria: SBP of less 
than $140 \mathrm{mmHg}$ and DBP of less than $90 \mathrm{mmHg}$ and no previous diagnosis with $\mathrm{EH}$.

The subjects were seated in a quiet room and prevented from smoking, exercising or drinking alcohol, tea or coffee for at least $1 \mathrm{~h}$ before the physical examination. The following data were recorded for each subject: name, age, gender, ethnicity, height, weight, body mass index (BMI), history of drinking and tobacco use (smoking was defined as smoking at least one cigarette per day for at least one year, and drinking was defined as consuming $50 \mathrm{~g}$ or more alcohol per day for at least one year) and blood pressure (SBP and DBP). Blood pressure was measured three times, with a 2-min interval between each measurement. SBP was recorded to the nearest $2 \mathrm{mmHg}$ at the appearance of the first Korotkoff sound (phase I), and DBP was recorded to the nearest $2 \mathrm{mmHg}$ at the disappearance of the fifth Korotkoff sound (phase V). The SBP and DBP values were calculated as the means of three consecutive physician-obtained measurements. Body weight and height were measured with subjects wearing only light indoor clothing and no shoes. BMI was calculated by dividing weight $(\mathrm{kg})$ by height squared $\left(\mathrm{m}^{2}\right)$. Blood samples were collected after an overnight fast, and total plasma cholesterol (TC), triglycerides (TG), high-density lipoprotein cholesterol (HDL-C), and low-density lipoprotein cholesterol (LDL-C) were measured within $8 \mathrm{~h}$ in a local hospital. Informed consent was obtained from all subjects.

\section{Laboratory assays}

In this study, we chose nine tagSNPs of TSC (rs6499858, rs2289119, rs13306673, rs2010501, rs7188995, rs7204044, rs4784733, rs8063406, and rs2304478). Tagging SNPs were selected from the Chinese HapMap database (http://www.hapmap.org) based on pairwise $\mathrm{r}^{2} \geq 0.5$ among all 1041 common SNPs with minor allele frequency $\geq 0.05$, as described elsewhere (Carlson et al., 2004).

Genomic DNA was extracted from peripheral blood leukocytes using a commercial blood DNA extraction kit (Genomic DNA purification kit; TaKaRa Biotechnology, Dalian, China) and was stored at $-20^{\circ} \mathrm{C}$. All nine tagSNPs of $T S C$ were genotyped using the polymerase chain reaction (PCR)/ligase detection reaction assay (Xiao et al., 2006). Primers were synthesized by Shanghai Sangon Biological Engineering Technology \& Services. Each set of ligase detection reaction probes comprised one common probe and two discriminating probes for the two types.

The target DNA sequences were amplified using a multiplex PCR method. PCRs for each subject were carried out in a final volume of $10 \mu \mathrm{L}$, containing $1 \mathrm{X}$ PCR buffer, $3.0 \mathrm{mM} \mathrm{MgCl}$, $2.0 \mathrm{mM}$ deoxynucleotide triphosphate, $0.4 \mu \mathrm{L}$ primers, $0.2 \mu \mathrm{L}$ Qiagen HotStarTaq Polymerase (QIAGEN, Shenzhen, China), $4 \mu \mathrm{L} 1 \mathrm{X}$ Q-solution, and 10-20 ng genomic DNA. Thermal cycling was performed for rs6499858, rs2289119, rs13306673, rs2010501, rs7188995, rs7204044, and rs4784733 in Gene Amp PCR system 9600 (PerkinElmer, Waltham, MA, USA) with an initial denaturation of $15 \mathrm{~min}$ at $95^{\circ} \mathrm{C}$, followed by 35 cycles of denaturation at $94^{\circ} \mathrm{C}$ for $30 \mathrm{~s}$, annealing at $62^{\circ} \mathrm{C}$ for $1 \mathrm{~min}$, and extension at $72^{\circ} \mathrm{C}$ for $1 \mathrm{~min}$, followed by a final extension at $72^{\circ} \mathrm{C}$ for 7 $\mathrm{min}$. Next, the protocol for rs8063406 and rs 2304478 amplification consisted of an initial denaturation of $15 \mathrm{~min}$ at $95^{\circ} \mathrm{C}$, followed by 35 cycles of denaturation at $94^{\circ} \mathrm{C}$ for $30 \mathrm{~s}$, annealing at $59^{\circ} \mathrm{C}$ for $1 \mathrm{~min}$, and extension at $72^{\circ} \mathrm{C}$ for $1 \mathrm{~min}$, followed by a final extension at $72^{\circ} \mathrm{C}$ for $7 \mathrm{~min}$.

The ligation reaction for each subject was carried out in a final volume of $10 \mu \mathrm{L}$, containing $1 X$ NEB Taq DNA ligase buffer, 12.5 pmol of each probe mix, $0.05 \mu \mathrm{L}$ Taq DNA ligase (NEB Biotechnology, Beijing, China), and $1 \mu \mathrm{L}$ multi-PCR product. A total of 35 cycles for ligase detection reaction were performed with $95^{\circ} \mathrm{C}$ for $2 \mathrm{~min}, 94^{\circ} \mathrm{C}$ for $30 \mathrm{~s}$, and $60^{\circ} \mathrm{C}$ for $2 \mathrm{~min}$. The fluorescent products of ligase detection reaction were differentiated by $\mathrm{ABI}$ 
sequencer 377 (Applied Biosystems, Foster City, CA, USA).

\section{Statistical analysis}

The comparison of clinical characteristics between the EH group and controls was performed using the Wilcoxon rank test, and the results are reported as median \pm interquartile range. The deviation from Hardy-Weinberg equilibrium for the variants was determined using the chi-square test. Allele and genotype frequencies between groups were compared using the chi-square test. Associations between genotypes and hypertension category were determined using unconditional logistic regression. A P value $<0.05$ was considered to be significant. Statistical analyses were performed using the SPSS software (version 13.0; SPSS, Chicago, IL, USA). We used the SHEsis software (Shi and He, 2005) to calculate the coefficient D' of linkage disequilibrium (LD) and to construct haplotypes. Haplotypes with frequencies $<3 \%$ in the whole sample were considered to be rare. There was a strong LD when D' $>0.8$.

\section{RESULTS}

\section{Clinical characteristics of EH and control subjects}

The parameters SBP, DBP, age, BMI, TG, HDL-C, and smoking (\%) were significantly higher in the EH group compared to controls in both the Mongolian and Han populations (Table 1); TC, LDL-C and drinking (\%) were markedly higher in the EH group than in controls in the Mongolian population.

\begin{tabular}{|c|c|c|c|c|c|c|}
\hline & \multicolumn{2}{|c|}{ Mongolians } & \multirow[t]{2}{*}{$\mathrm{P}$} & \multicolumn{2}{|c|}{ Han } & \multirow[t]{2}{*}{$\mathrm{P}$} \\
\hline & $\mathrm{NT}(\mathrm{N}=227)$ & $\mathrm{HT}(\mathrm{N}=158)$ & & $\mathrm{NT}(\mathrm{N}=239)$ & $\mathrm{HT}(\mathrm{N}=284)$ & \\
\hline Male/female & $97 / 130$ & $72 / 86$ & 0.068 & $142 / 97$ & $180 / 104$ & 0.203 \\
\hline Age (years) & $42.00 \pm 16.00$ & $51.00 \pm 18.00$ & $0.000 * *$ & $44.00 \pm 13.00$ & $49.00 \pm 15.50$ & $0.000 * *$ \\
\hline BMI $\left(\mathrm{kg} / \mathrm{m}^{2}\right)$ & $22.54 \pm 5.47$ & $24.76 \pm 4.43$ & $0.000 * *$ & $23.23 \pm 3.89$ & $25.06 \pm 3.35$ & $0.000 * *$ \\
\hline $\mathrm{SBP}(\mathrm{mmHg})$ & $109.06 \pm 12.32$ & $160.33 \pm 26.57$ & $0.000 * *$ & $119.44 \pm 11.42$ & $146.77 \pm 18.03$ & $0.000 * *$ \\
\hline $\mathrm{DBP}(\mathrm{mmHg})$ & $71.44 \pm 9.01$ & $104.51 \pm 14.67$ & $0.000 * *$ & $75.04 \pm 8.44$ & $90.20 \pm 12.37$ & $0.003 * *$ \\
\hline HDL-C (mM) & $1.54 \pm 0.66$ & $1.46 \pm 0.94$ & $0.046 *$ & $1.37 \pm 0.97$ & $1.24 \pm 0.36$ & $0.003 * *$ \\
\hline $\mathrm{TG}(\mathrm{mM})$ & $1.21 \pm 1.01$ & $1.87 \pm 1.27$ & $0.000 * *$ & $3.55 \pm 3.07$ & $1.58 \pm 1.63$ & $0.000 * *$ \\
\hline $\mathrm{TC}(\mathrm{mM})$ & $3.97 \pm 1.47$ & $5.33 \pm 2.32$ & $0.000 * *$ & $4.98 \pm 1.16$ & $4.91 \pm 1.19$ & 0.110 \\
\hline LDL-C (mM) & $2.45 \pm 1.02$ & $3.46 \pm 2.02$ & $0.000 * *$ & $3.07 \pm 0.87$ & $3.03 \pm 1.03$ & 0.088 \\
\hline Smoking (No/Yes) & $210 / 17$ & $106 / 52$ & $0.000 * *$ & $155 / 84$ & $221 / 63$ & $0.030^{*}$ \\
\hline Drinking $(\mathrm{No} / \mathrm{Yes})$ & $208 / 19$ & $60 / 98$ & $0.000 * *$ & $161 / 78$ & $194 / 90$ & 0.746 \\
\hline
\end{tabular}

Data are reported as median \pm interquartile range or as number of individuals. $\mathrm{BMI}=$ body mass index; $\mathrm{SBP}=$ systolic blood pressure; DBP = diastolic blood pressure; HDL-C = high-density lipoprotein-cholesterol; $\mathrm{TG}=$ triglycerides; $\mathrm{TC}=$ total cholesterol; $\mathrm{LDL}-\mathrm{C}=$ low-density lipoprotein-cholesterol. $* \mathrm{P}<0.05, * * \mathrm{P}<0.01$.

\section{Single-locus association study of tagSNPs and EH}

Genotype frequencies of all nine tagSNPs of TSC satisfied the Hardy-Weinberg equilibrium both in EH subjects and controls $(\mathrm{P}>0.05)$ in Mongolian and Han Chinese ethnic groups except for rs 8063406 .

The genotype distributions and allele frequencies are shown in Table 2. The distribu- 
tion of genotype and allele frequencies of rs7204044 and rs13306673 differed significantly between the EH group and controls in the Han population $(\mathrm{P}<0.05)$. The distribution of genotype and allele frequencies of rs 7204044 differed significantly between the EH group and controls in the Mongolian population $(\mathrm{P}<0.05)$.

The present study did not find any evidence for an association between rs6499858, rs2289119, rs2010501, rs7188995, rs4784733, rs8063406, or rs2304478 and hypertension. However, there were associations between the rs 7204044 variant and EH in Mongolian and Han Chinese ethnic groups in the additive model $(\mathrm{P}<0.05)$, and there were associations between the rs13306673 variant and $\mathrm{EH}$ in the Han population in the additive model $(\mathrm{P}<0.05)$.

\begin{tabular}{|c|c|c|c|c|c|c|c|c|c|}
\hline \multirow[t]{2}{*}{ Gene } & \multirow[t]{2}{*}{ Genotype/allele } & \multicolumn{4}{|c|}{ Han population } & \multicolumn{4}{|c|}{ Mongolian population } \\
\hline & & HT, N (\%) & NT, N (\%) & $\chi^{2}$ & $\mathrm{P}$ & HT, N (\%) & NT, N (\%) & $\chi^{2}$ & $\mathrm{P}$ \\
\hline \multirow[t]{5}{*}{ rs6499858 } & $\mathrm{CC}$ & $121(0.434)$ & $118(0.496)$ & 2.74 & 0.25 & $72(0.459)$ & $95(0.420)$ & 2.95 & 0.23 \\
\hline & $\mathrm{CT}$ & $128(0.459)$ & $102(0.429)$ & & & $75(0.478)$ & $105(0.465)$ & & \\
\hline & TT & $30(0.108)$ & $18(0.076)$ & & & $10(0.064)$ & $26(0.115)$ & & \\
\hline & $\mathrm{C}$ & $370(0.663)$ & $338(0.710)$ & 2.63 & 0.11 & $219(0.697)$ & $295(0.653)$ & 1.68 & 0.19 \\
\hline & $\mathrm{T}$ & $188(0.337)$ & $138(0.290)$ & & & $95(0.303)$ & $157(0.347)$ & & \\
\hline \multirow[t]{5}{*}{ rs2289119 } & AA & $21(0.075)$ & $19(0.080)$ & 0.33 & 0.85 & $17(0.108)$ & $18(0.082)$ & 1.15 & 0.56 \\
\hline & $\mathrm{AG}$ & $122(0.437)$ & $109(0.458)$ & & & $62(0.395)$ & $82(0.374)$ & & \\
\hline & GG & $136(0.487)$ & $110(0.462)$ & & & $78(0.497)$ & $119(0.543)$ & & \\
\hline & A & $164(0.294)$ & $147(0.309)$ & 0.27 & 0.60 & $96(0.306)$ & $118(0.269)$ & 1.19 & 0.28 \\
\hline & G & $394(0.706)$ & $329(0.691)$ & & & $218(0.694)$ & $320(0.731)$ & & \\
\hline \multirow[t]{5}{*}{ rs13306673 } & $\mathrm{CC}$ & $216(0.828)$ & $169(0.719)$ & 9.26 & $0.01 *$ & $121(0.834)$ & $179(0.825)$ & 0.39 & 0.82 \\
\hline & $\mathrm{CT}$ & $40(0.153)$ & $62(0.264)$ & & & $23(0.159)$ & $35(0.161)$ & & \\
\hline & TT & $5(0.019)$ & $5(0.019)$ & & & $1(0.007)$ & $3(0.014)$ & & \\
\hline & $\mathrm{C}$ & $472(0.904)$ & $400(0.851)$ & 6.57 & $0.01 *$ & $265(0.914)$ & $393(0.906)$ & 0.15 & 0.71 \\
\hline & $\mathrm{T}$ & $50(0.096)$ & $70(0.149)$ & & & $25(0.086)$ & $41(0.094)$ & & \\
\hline \multirow[t]{5}{*}{ rs2010501 } & $\mathrm{CC}$ & $16(0.057)$ & $12(0.051)$ & 0.77 & 0.68 & $7(0.045)$ & $11(0.049)$ & 0.12 & 0.90 \\
\hline & $\mathrm{CT}$ & $95(0.341)$ & $89(0.377)$ & & & $56(0.357)$ & $77(0.341)$ & & \\
\hline & TT & $168(0.602)$ & $135(0.572)$ & & & $94(0.599)$ & $138(0.611)$ & & \\
\hline & $\mathrm{C}$ & $127(0.228)$ & $113(0.239)$ & 0.20 & 0.66 & $70(0.223)$ & $99(0.219)$ & 0.02 & 0.90 \\
\hline & $\mathrm{T}$ & $431(0.772)$ & $359(0.761)$ & & & $244(0.777)$ & $353(0.781)$ & & \\
\hline \multirow[t]{5}{*}{ rs7188995 } & TT & $259(0.966)$ & $220(0.944)$ & 1.43 & 0.23 & $143(0.935)$ & $208(0.941)$ & 0.11 & 0.95 \\
\hline & GT & $9(0.034)$ & $13(0.056)$ & & & $9(0.059)$ & $12(0.054)$ & & \\
\hline & GG & 0 & 0 & & & $1(0.007)$ & $1(0.005)$ & & \\
\hline & $\mathrm{T}$ & $527(0.983)$ & $453(0.972)$ & 1.46 & 0.23 & $11(0.036)$ & $14(0.032)$ & 0.10 & 0.75 \\
\hline & $\mathrm{G}$ & $9(0.017)$ & $13(0.028)$ & & & $295(0.964)$ & $428(0.968)$ & & \\
\hline \multirow[t]{5}{*}{ rs7204044 } & AA & $6(0.021)$ & $4(0.017)$ & 7.01 & $0.03 *$ & $4(0.026)$ & $8(0.035)$ & 6.20 & $0.045^{*}$ \\
\hline & $\mathrm{AG}$ & $64(0.229)$ & $78(0.333)$ & & & $30(0.192)$ & $68(0.300)$ & & \\
\hline & GG & $210(0.750)$ & $152(0.650)$ & & & $122(0.782)$ & $151(0.665)$ & & \\
\hline & $\mathrm{A}$ & $76(0.136)$ & $86(0.184)$ & 4.43 & 0.04 & $38(0.122)$ & $84(0.185)$ & 5.52 & $0.018^{*}$ \\
\hline & $\mathrm{G}$ & $484(0.864)$ & $382(0.816)$ & & & $274(0.878)$ & $370(0.815)$ & & \\
\hline \multirow[t]{5}{*}{ rs4784733 } & $\mathrm{CC}$ & $6(0.024)$ & $7(0.031)$ & 0.27 & 0.87 & $4(0.028)$ & $3(0.014)$ & 1.99 & 0.37 \\
\hline & $\mathrm{CT}$ & $53(0.209)$ & $49(0.215)$ & & & $31(0.215)$ & $37(0.173)$ & & \\
\hline & TT & $195(0.768)$ & $172(0.754)$ & & & $109(0.757)$ & $174(0.813)$ & & \\
\hline & $\mathrm{C}$ & $65(0.128)$ & $63(0.138)$ & 0.92 & 0.64 & $39(0.135)$ & $43(0.100)$ & 2.07 & 0.15 \\
\hline & $\mathrm{T}$ & $443(0.872)$ & $393(0.862)$ & & & $249(0.865)$ & $385(0.900)$ & & \\
\hline \multirow[t]{5}{*}{ rs8063406 } & GG & $136(0.538)$ & $117(0.537)$ & 0.45 & 0.80 & $76(0.521)$ & $130(0.588)$ & 2.23 & 0.32 \\
\hline & GT & $30(0.119)$ & $30(0.138)$ & & & $19(0.130)$ & $20(0.090)$ & & \\
\hline & TT & $87(0.344)$ & $71(0.326)$ & & & $51(0.349)$ & $71(0.321)$ & & \\
\hline & $\mathrm{G}$ & $302(0.597)$ & $264(0.606)$ & 0.07 & 0.79 & $171(0.586)$ & $280(0.633)$ & 0.70 & 0.19 \\
\hline & $\mathrm{T}$ & $204(0.403)$ & $172(0.394)$ & & & $121(0.414)$ & $162(0.367)$ & & \\
\hline \multirow[t]{5}{*}{ rs 2304478} & AA & $2(0.008)$ & $2(0.009)$ & 2.47 & 0.29 & $3(0.021)$ & $2(0.009)$ & 2.45 & 0.29 \\
\hline & $\mathrm{AG}$ & $55(0.214)$ & $60(0.275)$ & & & $28(0.199)$ & $33(0.151)$ & & \\
\hline & GG & $200(0.778)$ & $156(0.716)$ & & & $110(0.780)$ & $184(0.840)$ & & \\
\hline & A & $59(0.115)$ & $64(0.147)$ & 2.14 & 0.14 & $34(0.121)$ & $37(0.084)$ & 2.51 & 0.11 \\
\hline & G & $455(0.885)$ & $372(0.853)$ & & & $248(0.879)$ & $401(0.916)$ & & \\
\hline
\end{tabular}

$\mathrm{HT}=$ hypertensives; $\mathrm{NT}=$ normotensives. $* \mathrm{P}<0.05$. 


\section{Haplotypes of tagSNPs}

The LD among the tagSNPs was measured by the Lewontin standardized disequilibrium coefficient D' in both groups separately (Slatkin, 2008). In the Mongolian population, three loci (rs2289119, rs13306673 and rs7204044) were found to be in strong LD (D'>0.8) (Table 3). In the Han population, three loci (rs7188995, rs7204044 and rs2304478) were found to be in strong LD (Table 4). Using the SHEsis software, we found that there are two haplotypes in the Han population and four haplotypes in the Mongolian population. The frequency of haplotype GCA in the EH group (16.6\%) was significantly higher than in the control group (9.4\%) in the Mongolian population. In the Han population the frequency of haplotype TGG was significantly higher in the EH group (75.2\%) than in controls $(66.5 \%)$, whereas haplotype TGA occurred significantly less often in EH (8.4\%) than in controls (12.5\%). Individuals who possessed the GCA haplotype had a significantly lower risk of EH in Mongolian, whereas the presence of haplotype TGG was significantly associated with a higher risk of EH in Han.

Table 3. TSC haplotype frequency distribution in the Mongolian population.

\begin{tabular}{lcrcrc}
\hline Haplotype & HT, N (\%) & NT, N (\%) & $\chi^{2}$ & OR & $95 \%$ CI \\
\hline ACG & $88.19(0.304)$ & $108.82(0.259)$ & 1.69 & 1.23 & $0.89-1.74$ \\
GCA & $27.31(0.094)$ & $69.55(0.166)$ & 7.54 & 0.52 & $0.33-0.84$ \\
GCG & $147.71(0.509)$ & $198.45(0.472)$ & 0.88 & 1.16 & $0.85-1.57$ \\
GTG & $21.08(0.073)$ & $33.73(0.080)$ & 0.15 & 0.90 & $0.51-1.58$ \\
Globe $\chi^{2}$ & 8.27 & & & & 0.348 \\
Fisher P & 0.04 & & & \\
\hline
\end{tabular}

HT = hypertensives; NT = normotensives; $\mathrm{OR}=$ odds ratio; $\mathrm{CI}=$ confidence interval; $\chi^{2}=$ Pearson's chi-square.

Table 4. TSC haplotype frequency distribution in the Han population.
\begin{tabular}{lcccccc}
\hline Haplotype & HT, N (\%) & NT, N (\%) & $\chi^{2}$ & OR & $95 \%$ CI & P \\
\hline TGA & $40.64(0.084)$ & $52.06(0.125)$ & 4.33 & 0.63 & $0.41-0.98$ & 0.038 \\
TGG & $362.36(0.752)$ & $276.54(0.665)$ & 7.42 & 1.53 & $1.13-2.09$ & 0.006 \\
Globe $\chi^{2}$ & 7.74 & & & & & \\
Fisher P & 0.02 & & & & & \\
\hline
\end{tabular}

$\mathrm{HT}=$ hypertensives; $\mathrm{NT}=$ normotensives; $\mathrm{OR}=$ odds ratio $; \mathrm{CI}=$ confidence interval; $\chi^{2}=$ Pearson's chi-square.

\section{DISCUSSION}

We examined the relationships between tagSNPs of TSC gene and hypertension in Mongolian and Han populations. By systemically screening variations of TSC and studying the associations of nine representative tagSNPs with hypertension, we identified associations between rs7204044, rs13306673 and hypertension in Han, and between rs7204044 and hypertension in the Mongolian population in Inner Mongolia.

It has been reported that several novel homozygous mutations of the TSC gene lead to loss of TSC function and thus reduced renal sodium reabsorption, as seen in Gitelman's syndrome (Simon et al., 1996; Fava et al., 2008; Miao et al., 2009). TSC, which is located in the distal renal tubules, is a target of the thiazide diuretics, which are known to be useful for patients with hypertension who are salt-sensitive (Peters and Flack, 2000). Melander et al. 
(2000) investigated TSC gene polymorphisms (G2736A, C1420T, G816C), and showed that homozygous $A 2736$ and $T 1420$ alleles were significantly associated with hypertension in the Swedish population. Matsuo et al. (2004) replicated the findings made by Melander et al. (2000). They found that $G 2736 \mathrm{~A}$ polymorphism of the TSC gene was a genetic predisposing factor for EH in Japanese women. Another study performed on a Canadian population failed to demonstrate a correlation between the TSC G2736A polymorphism and hypertension (Keszei et al., 2007). These findings indicate that the TSC gene may be involved in human hypertension. Analysis of a single-locus polymorphism has its limitations in correlation studies. To guard against this potential limitation we conducted a comprehensive association analysis of tagSNPs of TSC gene in Mongolian and Han ethnic groups in Inner Mongolia. Haplotype analysis was also performed.

In this study, we found that rs 7204044 and rs 13306673 of the TSC gene were genetic predisposing factors for EH in Han, and that rs7204044 was a genetic predisposing factor for EH in Mongolian in Inner Mongolia. The mechanisms by which rs7204044 and rs13306673 might contribute to hypertension are currently unknown. rs 7204044 and rs 13306673 are located in an intronic region. It has traditionally been assumed that there is no biological function of intron. However, introns contain several short sequences that are important for efficient splicing (Crosio et al., 1996). Therefore, further investigation is necessary to determine whether the polymorphisms of rs 7204044 and rs 13306673 affect the splicing of mRNA of the TSC gene. In addition, it remains possible that rs7204044 and rs13306673 are mere genetic markers, and it may be that LD with other functional variations within the TSC gene and other functional polymorphisms play more important roles in hypertension. Therefore, we studied the relationship between haplotypes of TSC and hypertension.

Haplotype analysis has more detection power than single polymorphisms in genetic association studies, because the interaction of multiple genetic markers within a haplotype could be a key factor for disease susceptibility rather than the individual polymorphism (Cardon and Abecasis, 2003; Jacob-Ferreira and Sandrim, 2007). Particularly if the markers used define mutations within functional DNA, then the haplotypes composed of these markers would be more likely to have a biological role. The identification of the haplotype associated with $\mathrm{EH}$ and the measures of LD are important in the investigation of genetic marker(s) of EH in Mongolian and Han Chinese populations. We found a strong LD in both ethnic groups. Furthermore, individuals who possessed the GCA haplotype had a lower risk of EH in Mongolian, whereas the presence of haplotype TGG was associated with a higher risk of EH in Han. Given diverse results of relationships with tagSNPs and haplotype in the two populations, we deduced that the differences in hypertension are related to predisposing factors in terms of lifestyle, physical activity and other socioeconomic factors that could affect blood pressure instead of genetic factors.

In conclusion, rs 7204044 of TSC is potentially a genetic factor for EH in these two ethnicities, rs 13306673 is potentially a genetic factor for EH in Han population, and a rare haplotype of the gene might be protective against the development of hypertension. These findings may help find ways to prevent and treat EH in Mongolian and Han Chinese populations in Inner Mongolia.

\section{ACKNOWLEDGMENTS}

We appreciate the support and assistance provided by the Duolun Center for Disease Prevention and Control and the Department of Epidemiology of the Inner Mongolia Medical School. 


\section{REFERENCES}

Cardon LR and Abecasis GR (2003). Using haplotype blocks to map human complex trait loci. Trends Genet. 19: 135-140.

Carlson CS, Eberle MA, Rieder MJ, Yi Q, et al. (2004). Selecting a maximally informative set of single-nucleotide polymorphisms for association analyses using linkage disequilibrium. Am. J. Hum. Genet. 74: 106-120.

Crosio C, Cecconi F, Mariottini P, Cesareni G, et al. (1996). Fugu intron oversize reveals the presence of U15 snoRNA coding sequences in some introns of the ribosomal protein S3 gene. Genome Res. 6: 1227-1231.

Fava C, Montagnana M, Rosberg L, Burri P, et al. (2008). Subjects heterozygous for genetic loss of function of the thiazide-sensitive cotransporter have reduced blood pressure. Hum. Mol. Genet. 17: 413-418.

Jacob-Ferreira AL and Sandrim VC (2007). Endothelial nitric oxide synthase polymorphisms and hypertension: improved clinical evidence derived from haplotype analysis. Int. J. Cardiol. 116: 116.

Keszei AP, Tisler A, Backx PH, Andrulis IL, et al. (2007). Molecular variants of the thiazide-sensitive $\mathrm{Na}^{+}-\mathrm{Cl}^{-}$cotransporter in hypertensive families. J. Hypertens. 25: 2074-2081.

Matsuo A, Katsuya T, Ishikawa K, Sugimoto K, et al. (2004). G2736A polymorphism of thiazide-sensitive Na-Cl cotransporter gene predisposes to hypertension in young women. J. Hypertens. 22: 2123-2127.

Melander O, Orho-Melander M, Bengtsson K, Lindblad U, et al. (2000). Genetic variants of thiazide-sensitive NaClcotransporter in Gitelman's syndrome and primary hypertension. Hypertension 36: 389-394.

Miao Z, Gao Y, Bindels RJ, Yu W, et al. (2009). Coexistence of normotensive primary aldosteronism in two patients with Gitelman's syndrome and novel thiazide-sensitive Na-Cl cotransporter mutations. Eur. J. Endocrinol. 161: 275-283.

Obermüller N, Bernstein P, Velazquez H, Reilly R, et al. (1995). Expression of the thiazide-sensitive Na-Cl cotransporter in rat and human kidney. Am. J. Physiol. 269: F900-F910.

Peters RM and Flack JM (2000). Salt sensitivity and hypertension in African Americans: implications for cardiovascular nurses. Prog. Cardiovasc. Nurs. 15: 138-144.

Plotkin MD, Kaplan MR, Verlander JW, Lee WS, et al. (1996). Localization of the thiazide sensitive Na-Cl cotransporter, rTSC1 in the rat kidney. Kidney Int. 50: 174-183.

Shi YY and He L (2005). SHEsis, a powerful software platform for analyses of linkage disequilibrium, haplotype construction, and genetic association at polymorphism loci. Cell Res. 15: 97-98.

Simon DB, Nelson-Williams C, Bia MJ, Ellison D, et al. (1996). Gitelman's variant of Bartter's syndrome, inherited hypokalaemic alkalosis, is caused by mutations in the thiazide-sensitive Na-Cl cotransporter. Nat. Genet. 12: 24-30.

Slatkin M (2008). Linkage disequilibrium - understanding the evolutionary past and mapping the medical future. Nat. Rev. Genet. 9: 477-485.

Wang XF, Lin RY, Wang SZ, Zhang LP, et al. (2008). Association study of variants in two ion-channel genes (TSC and CLCNKB) and hypertension in two ethnic groups in Northwest China. Clin. Chim. Acta 388: 95-98.

Xiao Z, Xiao J, Jiang Y, Zhang S, et al. (2006). A novel method based on ligase detection reaction for low abundant YIDD mutants detection in hepatitis B virus. Hepatol. Res. 34: 150-155.

Zhan YY, Jiang X, Lin G, Li J, et al. (2007). Association of thiazide-sensitive $\mathrm{Na}^{+}-\mathrm{Cl}^{-}$cotransporter gene polymorphisms with the risk of essential hypertension. Zhonghua Yi. Xue. Yi. Chuan Xue. Za Zhi. 24: 703-705. 